\title{
Consumo de marihuana y deterioro cognitivo en estudiantes universitarios, Pereira 2019
}

\author{
Anyi Marcela Mejía Silva', Ladis Geraldín Cuaran Silva², \\ Luisa Fernanda Esquivel Holguín³, Germán Oved Acevedo Osorio4, \\ William Duque Salazar ${ }^{5}$.
}

RESUMEN Introducción: el consumo de cannabis se presenta como una problemática de salud pública, la cual perjudica a toda la sociedad, pero en especial a los jóvenes que inician esta actividad desde temprana edad, dado que tienen una perspectiva del cannabis como una droga blanda; así mismo responde a una baja percepción de riesgo, fundamentada en creencias erróneas e influenciada por factores individuales, microsociales, macrosociales y variables demográficas. Las drogas no solo conllevan a una adicción, sino que, además, alteran las manifestaciones cognitivas de la conducta, incluyendo la memoria, la emoción, la atención y la toma de decisiones, con pérdida de control importante, de tal modo que genera un deterioro cognitivo significativo y posiblemente relacionado a un bajo desempeño académico.

PALABRAS Consumo;

CLAVE $\quad$ Deterioro Cognitivo;

Pérdida de Memoria

1 Estudiante, Fundación Universitaria del Área Andina, Semillero de investigación Los Asépticos.amejia51@estudiantes.areandina.edu.co

2 Estudiante, Fundación Universitaria del Área Andina, Semillero de investigación Los Asépticos. Icuaran@estudiantes.areandina.edu.co

3 Estudiante, Fundación Universitaria del Área Andina, Semillero de investigación Los Asépticos. lesquivel2@estudiantes.areandina.edu.co

4 Asesor, Docente, Fundación Universitaria del Área Andina, Grupo de investigación Los Asépticos.gacevedo@areandina.edu.co

5 Asesor, Docente, Fundación Universitaria del Área Andina, Grupo de investigación Los Asépticos.wduque@areandina.edu.co 


\title{
Marïuana consumption and cognitive impairment in university students, Pereira 201
}

\begin{abstract}
Introduction: cannabis use is presented as a public health problem, which harms society as a whole, but especially young people who start this activity from an early age, given that they have a perspective on cannabis as a soft drug; it also responds to a low perception of risk, based on mistaken beliefs and influenced by individual factors, microsocial, macrosocial and demographic variables. Drugs not only lead to addiction, but also alter cognitive behavioral manifestations, including memory, emotion, attention, and decision-making, with significant loss of control, so that it generates significant cognitive impairment and possibly related to low academic performance.
\end{abstract}

\section{Cannabis;}

KEYWORDS Use;

Cognitive Impairment;

Memory Loss 


\section{INTRODUCCIÓN}

La oficina de las Naciones Unidas Contra la Droga y el Delito (UNODC), hace un seguimiento al consumo de drogas, donde según un reporte publicado en el 2017, más de 250 millones de personas han probado sustancias psicoactivas y por lo menos 29.5 de millones de consumidores presentaron trastornos vinculados con drogas (1); por tal motivo la drogadicción, en especial el consumo de marihuana, es considerado un problema de salud pública, debido a que es la sustancia psicoactiva ilegal con mayor consumo alrededor del mundo (2).

Colombia ha tenido un aumento significativo con relación al consumo de marihuana, donde múltiples estudios han podido evidenciar un inicio temprano y prolongado entre los 12 a 65 años, de los cuales el 11,5\% ha consumido marihuana alguna vez en su vida (3). Según un estudio realizado en el 2014 en la Universidad Pública de Norte de Santander en estudiantes de primer semestre, se presentó una prevalencia del $7,1 \%$ entre las edades de 18 a 25 años, donde igualmente se analizaron las variables sociodemográficas para conocer los factores que conllevan al consumo de cannabis, en donde se observó que el género masculino tiene una prevalencia superior del $8,7 \%$ con respecto al género femenino, el cual es de 4,4\%. (2).

El consumo de marihuana se encuentra asociado al deterioro cognitivo, físico y en especial social (2), cuyos efectos varían dependiendo de su concentración y del Fito cannabinoides, pues existen diversos prototipos y cada uno tiene una farmacodinamia diferente (3), los cuales se encuentran relacionados con la pérdida de la memoria, alteraciones en el aprendizaje y además pérdida del control interno (2).

Esta problemática conlleva a un punto crítico, el cual es generado por el poco autocontrol del consumo, de manera que trae consigo efectos cognitivos de importancia, por eso, la Organización Mundial de la Salud (OMS) toma la marihuana como una sustancia que tiene la capacidad de cambiar la conciencia, estado emocional o procesos del pensamiento en un individuo, debido a que el cannabis puede afectar al cerebro de manera estructural, pero que la población no ha considerado sus efectos relacionados al deterioro desde las distintas perspectivas psicológicas, fisiológicas y sociales, por desconocimiento y cultura de consumo actual (3).

La interacción entre el consumo y el sistema nervioso central inicia con los astrocitos; es decir entre los vasos sanguíneos y las neuronas, allí llegan los cannabinoides donde distorsionan, ya sea aumentando o disminuyendo el suministro de energía a las neuronas; de esta manera se puede afectar una función principal como lo es el metabolismo y en sí la transmisión neuronal, además interactúa con los neurotransmisores afectando los comportamientos fisiológicos (4).

Teniendo en cuenta que existen varios tipos de cannabinoides, uno de los más importantes es el tipo 1 (CB1) que influye de manera excesiva en la transmisión 
neuronal, funcional y plasticidad, puesto que transforma al cerebro e influye en la expresión del lenguaje; los astrocitos contienen al CB1 que ayudan a controlar las respuestas inflamatorias, sin embargo, pueden controlar la comunicación de la neurona con el astrocito; incluso existe otro Fito cannabinoides como el CB-LTD el cual en el hipocampo genera deterioro en la memoria, de manera que no solo afecta la parte del sistema nervioso central sino que de igual forma el sistema endocrino, ya que al alterar por medio del SNC el metabolismo, genera la producción de diversas hormonas que con el tiempo producen ansiedad y se refleja obesidad (4).

Lo anterior es causado también porque en el hipotálamo se aumenta la proteína ácida fibrilar glial (GFAP) debido al CB-LTD, los endocannabinoides afectan no solo el balance energético, sino que los niveles al interactuar los sistemas neurotransmisores, neuropéptidos y hormonas como lo es la leptina, que cumple un papel importante: regular el balance de energía al SNC. (4) Por otra parte la Organización Panamericana de la Salud (OPS), afirma que cuando se consume cualquier droga se libera un neurotransmisor, dopamina en el Núcleo de Accumbens, y si se hace en exceso afecta funciones emocionales, la planificación de la conducta, el aprendizaje y la memoria, pero en especial genera adicción.

En lo que respecta al consumir marihuana, se genera un deterioro cognitivo posiblemente asociado a un bajo rendimiento académico, dado a la poca capacidad de memorizar o acordarse de cosas importantes, también influye en la disminución a integraciones grupales, que afectan su vida social. En definitiva, el cannabis desestabiliza la morfofisiología, afectando partes del cerebro, las cuales se ven alteradas por una sobredosis; por otro lado, en los programas de concientización frente al consumo de cannabis, se puede observar una gran brecha con la información sobre los efectos cognitivos producidos por su consumo rutinario, debido a que la población juvenil considera que las consecuencias derivadas son mínimas o leves (3).

La OPS prioriza el consumo en jóvenes adultos entre los 15 y 25 años, que es la edad en la cual se encuentran los estudiantes universitarios, asociado a débiles programas frente a la concientización de los efectos negativos de la marihuana, que podrían repercutir de manera positiva en los procesos de aprendizaje-enseñanza si se realiza un trabajo interdisciplinario con el enfoque educativo pertinente.

Por los motivos anteriormente expuestos, se plantea la siguiente pregunta de investigación ¿Cuál es el nivel de deterioro cognitivo en una muestra de estudiantes universitarios consumidores frecuentes de marihuana del municipio de Pereira, en el año 2019?

\section{METODOLOGÍA}

Tipo de estudio: observacional, descriptivo y transversal.

Población y muestra: la población está conformada por estudiantes universitarios 
con consumo frecuente de cannabinoides, de una Institución de Educación Superior IES, en el año 2019.

Criterios de inclusión: estudiantes mayores de edad matriculados legal y financieramente en el semestre 2019-3, que deseen participar en el estudio y cumplan con los criterios de consumidores frecuentes del test de CAST.

Criterios Exclusión: consumidores ocasionales.

Recolección de información: para la recolección de información se utilizará el test de CAST, el cual nos permite conocer la frecuencia de uso de marihuana y además identificar situaciones problemáticas; el test de Neuropsi evalúa las funciones cognoscitivas que comprende áreas como concentración, memoria, orientación, lenguaje, habilidades viso-espaciales, funciones ejecutivas y de lectoescritura, además se utilizarán variables sociodemográficas, y por último se implementarán las variables que se encuentran en el artículo de ilusión cognitiva de Galván G ET. AL. 2017.

Tabulación de datos: los datos serán tabulados en el programa informático Microsoft Office Excel 2017®.

Análisis de la información: la información será analizada en SPSS versión 23. La variable cualitativa se describirá mediante tablas de frecuencias (absolutas, relativas). Para las variables cuantitativas se utilizarán medidas de tendencia central y medidas de dispersión. Para el análisis bivariado se utilizará la prueba Chi-cuadrado de Pearson, para variables cualitativas. Para análisis cuantitativo se aplicarán pruebas paramétricas y no paramétricas, según corresponda su distribución.

De acuerdo de la Resolución 008430 de 1993 y al Comité de Investigación del programa de Instrumentación Quirúrgica, este estudio se considera sin riesgo, por lo cual es suficiente garantizar la confidencialidad de la información y participación voluntaria en el estudio, con la previa firma del consentimiento informado.

Compromiso o referente bioético: de acuerdo con la Resolución 0008430 de 1993, el estudio se considera sin riesgo. De igual manera se aplicará el instrumento con previa firma del consentimiento informado.

\section{RESULTADOS ESPERADOS}

- Se espera poder establecer la frecuencia del consumo y su nivel con el deterioro cognitivo en estudiantes universitarios, asociado a un posible bajo desempeño académico, con el fin de concientizar a la población juvenil sobre las consecuencias del consumo de cannabis. 
- Se espera divulgar los resultados en eventos nacionales e internacionales, con publicación en revistas indexadas en el área de interés.

REFERENCIAS 1. Banchero LA, Canseco-Carrasco Carlos D, Espinosa-Bedoya O, Royle HF, Boehm FR, Roman-Morey E, et al. El Problema de las Drogas en el Perú 2018. 1.a ed. Perù: CEDRO; 2018. 1-217 p.

2. Martinez-Torres J, Arias-Coronel F, Rodelo-Vega AE, Jaraba-Toro NP, MezaCastellanos LM, Contreras-Jáuregui MM, et al. Prevalencia y factores asociados al consumo de marihuana en estudiantes de 18 a 25 años de una universidad pública, Colombia. Rev. Udenar. 2016;18(3):525-31.

3. Galván G, Guerrero-Martelo M, Vásquez De La Hoz F. Artículo original Cannabis: una ilusión cognitiva. Rev. Colomb Psiquiatr. 2017;46(2):96-102.

4. Bosier B, Bellocchio L, Metna-Laurent M, Soria-Gómez E, Matías I, HebertChatelain E, et al. Astroglial CB 1 cannabinoid receptors regulate leptin signaling in mouse brain astrocytes. Mol Metab. 2013;2(August):393-404. 